\title{
A review of studies establishing that keyword method effects are robust with respect to temporal characteristics of presentation
}

\author{
MICHAEL PRESSLEY \\ University of Western Ontario, London, Ontario, Canada
}

\begin{abstract}
Since $1981 \mathrm{Hall}$ and his associates have argued that the presentation of to-be-learned vocabulary items in keyword experiments has been biased to favor keyword over control subjects. Hall, Owens, and Wilson (1987) claimed that many keyword experiments involved single, slow presentations. According to Hall et al., since control learning is better with multiple, rapid presentations than with single, slow presentations (i.e., there is a distributed practice effect with control instructions), control subjects in the previous experiments were presumably handicapped. The critical question, as posed by Hall and his coworkers since 1981, is whether there are presentation method $\times$ instructional (keyword vs. no-instruction control) interactions, such that keyword effects are larger with single, slow presentations of vocabulary than with presentations having other temporal characteristics (e.g., free study of all items simultaneously; multiple, rapid presentations). Four unconfounded tests of such interactions have been reported in the literature to date. All suggest that keyword method effects are robust with respect to temporal presentation variables. The relevant data are reviewed here.
\end{abstract}

In a recent article, Hall, Owens, and Wilson (1987) argued that keyword method research has been characterized by a bias that favors keyword conditions over control conditions due to slow presentation of the to-be-learned vocabulary items. This is the latest instantiation of a general argument made throughout the 1980s by Hall and his associates (e.g., Hall, Wilson, \& Patterson, 1981) that temporal characteristics of item presentations in keyword studies subtly favor keyword over control subjects, at least when sophisticated learners such as university students are subjects. Hall et al. (1987) are certainly correct that many keyword experiments have involved slow-paced presentation of vocabulary (e.g., $10 \mathrm{sec}$ per item, one word presented at a time). Nonetheless, results of other experiments that were generated in response to Hall's previously published criticisms permit the conclusion that positive keyword method effects are probably robust with respect to pace-of-presentation characteristics. The main purpose of this article is to alert readers to these studies.

I begin with the only relevant interaction data in the literature that could be construed as support for Hall et al.'s (1981; Hall et al., 1987) contention that keyword method effects occur given some presentation formats but not others. When university students in Hall et al.'s (1981) Experiment 4 were given Spanish words to study, they either were told to use the keyword method or were left

Preparation of this article was supported by a grant to the author from the Natural Sciences and Engineering Research Council of Canada. Correspondence about the article can be addressed to the author at the Department of Psychology, University of Western Ontario, London, ON N6A 5C2, Canada. to their own devices. Subjects were presented with the words in one of two pacing formats, either one word every $10 \mathrm{sec}$ (paced study) or the entire list of to-be-acquired vocabulary for the full study period (free study). There was a main effect of pacing: free study produced better learning than paced presentation. More critically, there was a significant interaction in that control recall was greater than keyword recall with free study, and keyword recall was superior to control recall with paced presentation. Based on these data, Hall et al. (1981) reasoned that the spontaneous learning strategies of uninstructed university students were presumably impaired by paced presentation of the to-be-learned vocabulary, and that, therefore, a paced, control condition was not challenging. As they stated, "the keyword method might be a powerful enough study strategy to prove superior when self-generated strategies are restricted by rigidly paced presentation but not so powerful as strategies generated when bright students are given greater control over their study activities" (p. 356).

In reviewing Hall et al.'s (1981) Experiment 4, I noted, however, a complete confounding of pacing during studying and testing (i.e., paced study subjects were given a paced test, and free study subjects were given all of the items simultaneously on their test). I also noted that Hall et al. (1981, Experiment 4) had not replicated the large, statistically significant $(p<.05)$ keyword effect under paced conditions, a finding replicated many times in the literature (e.g., Pressley, Levin, \& Delaney, 1982), but instead had obtained a trend. Thus, Pressley, Levin, Digdon, Bryant, and Ray (1983) conducted two experiments with university students as subjects in which the studying-testing confounding in Hall et al. (1981) was 
eliminated. Pacing varied only at study (paced vs. free study) in no-instruction control and keyword conditions. Particularly important, Pressley et al. (1983) employed keyword instructions that had been shown previously to produce unambiguous gains when vocabulary items were presented in a paced fashion. Only main effects were detected in Pressley et al.'s (1983) study. The keyword method instructions produced better learning than did control instructions. Free study produced significantly better learning than did paced study in one of the experiments, and a trend in the same direction in the other study. Most critically, nothing in these data suggested that keyword method effects were confined to the slow-paced presentation condition.

As part of a larger investigation, McCormick, Miller, and Fields (1987) also included an examination of the potential interaction proposed by Hall et al. (1981). The results were consistent with Pressley et al.'s (1983) study, with main effects for keyword over control and for free study over paced presentation, but no interaction between these variables. In short, the three experiments that provided unconfounded tests of paced presentation versus free study failed to support the hypothesis that keyword method effects are limited to paced situations.

The two experiments reported by Hall et al. (1987) were described and discussed previously by $\mathrm{Hall}$ and $\mathrm{Fu}-$ son (1986). The argument that keyword method effects may be limited to particular presentation formats is given a slightly different focus in these two articles, compared with the claims made by Hall et al. (1981). Hall and his associates demonstrated a distributed practice effect on vocabulary learning (Hall et al., 1987, Experiment 1). Based on this finding, they claimed that control subjects in keyword studies who received vocabulary in one massed presentation and at a slow rate were possibly disadvantaged, because learning under control instructions is more efficient given several short presentations than given one massed presentation. Hall and Fuson (1986) pointed to the data subsequently reported by Hall et al. (1987, Experiment 2) as support for this interpretation.

I found Hall and Fuson's (1986) argument strained. A test of this new pacing $\times$ condition interaction hypothesis would minimally require single-, slow-presentation control and keyword conditions, as well as multiple-, rapid-presentation control and keyword conditions. Hall et al. (1987, Experiment 2) included only three of the four necessary conditions, omitting the multiple-, rapidpresentation keyword condition. ${ }^{1}$ In short, the design of Experiment 2 presented by Hall et al. (1987) could not possibly produce information about whether keyword method effects are confined to slow, single presentations.

Hall and his associates' rationale for this incomplete design was that he and his colleagues had tested 5 subjects in a multiple-, rapid-presentation keyword condition (as part of Hall et al., 1987, Experiment 1), and all of the subjects had complained that they could not execute the keyword method given the rapid-fire setup. One in- terpretation of these complaints is that there might have been a problem with Hall et al.'s (1987) keyword directions, a hunch supported by their failure to obtain the classic, slow-presentation keyword effect consistently in their Experiment 2; there were three keyword versus control comparisons in Hall et al.'s (1987) Experiment 2, with only one of them favoring the keyword method.

Even though Hall and his colleagues had no convincing data to support their claim, I considered the hypothesis presented by Hall and Fuson (1986) to be plausible and one that deserved attention in the vocabulary literature if true. I also surmised, however, that the keyword instructions that had been highly effective in my own previous research might be sufficient to induce keyword use with multiple, rapid presentations. If that were the case, it might be possible to add the effects produced by multiple, rapid presentations and the keyword method to produce an especially powerful intervention.

Thus, I conducted a test of Hall and Fuson's (1986) interaction hypothesis, again using keyword instructional methods previously demonstrated to produce large effects when vocabulary items are presented one at a time at a slow rate. As previously described (Pressley, 1987), university students were presented 35 Latin words to learn and either were instructed to use the keyword method to study or were left to their own devices to acquire the items. Half of the subjects in each of the two instructional conditions were presented each vocabulary item one time for $9 \mathrm{sec}$ (corresponding to Hall et al.'s, 1987, $1 \times 9$ condition), and half were presented each word three times for $3 \mathrm{sec}$ (corresponding to Hall et al.'s, 1987, $3 \times 3$ condition). In contrast to Hall et al.'s (1987) difficulties in getting university students to execute the keyword method given rapid presentation, none of Pressley's (1987) keyword subjects reported difficulty executing the procedure, with this conclusion supported by detailed postexperiment interviews about mediation that occurred during study. There were two main effects: keyword subjects learned more than controls, and $3 \times 3$ subjects learned more than $1 \times 9$ subjects. There was no instructional method $\times$ presentation method interaction.

In summary, I know of four unconfounded tests of the hypothesis put forward by Hall and his colleagues that keyword method effects are more certain with slow, paced presentations of vocabulary than with other presentation methods. None provided support for the interaction hypothesis.

Those who might continue the search for possible presentation method $\times$ instructional condition interactions involving the keyword method should pay special attention to one important methodological point. The necessary starting point for such research is convincing demonstration that the keyword instructions in use actually produce keyword method mediation as defined in the literature. Adequate keyword method use can be inferred from on-line self-reports and in-depth postexperiment interviews. Especially convincing evidence that effective key- 
word method instructions are in place, however, is replication of the now classic single-, slow-presentation keyword effect as part of the overall test of interaction. See Pressley and McDaniel (in press) for more extensive commentary about methodology in keyword method studies; McDaniel and Pressley (1987) provide up-to-date reviews about keyword method research.

I would be remiss if I did not point out that Hall's research and writing have had two important and positive impacts. Obviously, Hall and his associates have stimulated investigation of the keyword method using alternative presentation methods. This is good, because the proportion of keyword studies that included only slow, paced presentation of vocabulary is disturbingly high. Second, Hall's work has also brought into focus the fact that presentation methods alone can be used to facilitate vocabulary learning. I have had no difficulty replicating the free study versus pacing and the spaced presentation versus massed presentation effects that Hall and his research group have identified. Dempster (1987) provided additional data confirming the distributed practice effect in vocabulary learning. The consistency of these presentation method main effects makes obvious that they should be taken into account in designing vocabulary learning interventions. Additional work on these and other presentation formats could be valuable to the extent that it can be tied convincingly to important theoretical and/or educational issues.

\section{REFERENCES}

DEMPSTER, F. N. (1987). Effects of variable encoding and spaced presentation on vocabulary learning. Journal of Educational Psychology, 79, 162-170.

Hall, J. W., \& Fuson, K. C. (1986). Presentation rates in experiments on mnemonics. Journal of Educational Psychology, 78, 233-234.
Hall, J. W., Owens, W. L., \& Wilson, K. P. (1987). Presentation rates and keywords in vocabulary learning. Bulletin of the Psychonomic Society, 25, 179-181.

Hall, J. W., Wilson, K. P., \& Patterson, R. J. (1981). Mnemotechnics: Some limitations of the mnemonic keyword method for the study of foreign language vocabulary. Journal of Educational Psychology, 73, 345-357.

McCormick, C. B., Miller, G., \& Fields, J. T. (1987, April). A comparison of contextual and mnemonic approaches to learning English vocabulary: The effects of pacing. Presented at the annual meeting of the American Educational Research Association, Washington, DC.

McDaniel, M. A., \& Pressley, M. (1987). Imagery and related mnemonic processes: Theories, individual differences, and applications. New York, NY: Springer-Verlag.

Pressley, M. (1987). Are keyword method effects limited to slow presentation rates? An empirically-based reply to Hall and Fuson (1986). Journal of Educational Psychology, 79, 333-335.

Pressley, M., Levin, J. R., \& Delaney, H. D. (1982). The mnemonic keyword method. Review of Educational Research, 52, 61-91.

Pressley, M., Levin, J. R., Digdon, N., Bryant, S. L., \& Ray, K. (1983). Does method of item presentation affect keyword method effectiveness? Journal of Educational Psychology, 75, 686-691.

Pressley, M., \& MCDaniel, M. A. (in press). Doing mnemonics research well: Some general guidelines and a study. In M. Gruneberg, P. Morris, \& R. N. Sykes (Eds.), Practical aspects of memory. London, England: Wiley.

\section{NOTE}

1. Hall and Fuson (1986) described a still unpublished experiment that nominally included the four necessary conditions. Unfortunately, the control and keyword conditions in that experiment were completely confounded with amount of in-the-experiment practice in vocabulary learning. That is, control learning was assessed before there was any practice learning vocabulary, whereas keyword method performance was assessed after 4 weeks of instruction with vocabulary learning practice. 